\title{
NOTES AND CORRESPONDENCE A Note on the Stability of the Tropical Easterlies
}

\author{
By S. G. H. Philander \\ Geophysical Fluid Dynamics Programme* \\ Princeton University, Princeton, New Jersey 08540 \\ (Manuscript received 1 March 1976, in revised form 24 August 1976)
}

\section{Introduction}

Nitta and Yanai (1969) showed that only on some occasions did the observed easterly winds in the neighborhood of the Marshall Islands satisfy the necessary condition for barotropic instability, namely that the potential vorticity gradient $\left(\beta-U_{y y}\right)$ changes sign.

(The latitudinal profile of the winds is described by $U(y)$ where $y$ measures distance in a northward direction.) They concluded that barotropic instability of the easterlies could be a weak mechanism for the generation of the disturbances with a period of 4 to 5 days observed along the Intertropical Convergence Zone. It is the purpose of this note to point out that the necessary condition for instability (1) applies to non-divergent flow, and may be too stringent if the effects of divergence are taken into account. Lipps' (1963) study of the effects of divergence on barotropic instability is extended to cases of arbitrarily large

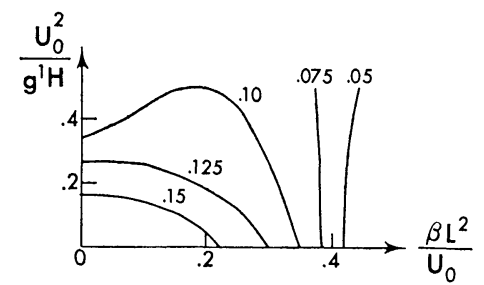

Fig. 1 Contours of constant growth-rate (in units $\left.U_{o} / L\right)$ as a function of the $\beta$ effect (as measured by the parameter $\beta L^{2} / U_{o}$ ) and divergence (as measured by the parameter $\left.U_{o}^{2} / g^{\prime} H\right)$ for wavelength $4 \pi L$, for the shear flow $U=$ $-U_{o} \tan h y / L$ centered on the equator. This wavelength is, for practical purposes, the most unstable one. (See Philander 1976).

\footnotetext{
* Support provided through Geophysical Fluid Dynamics Laboratory/NOAA Grant No. 04-3-022-33.
}

growth rates. A linear but ageostrophic model is used so that equatorial winds can be studied. (For a detailed description of the model, and of the general results, see Philander (1976). This note concerns only the meteorological implications of the results.)

The importance of divergence is found to depend on the latitudinal profile of the mean winds. We consider two examples: winds with a shearlayer-like structure, such as the easterlies over the Marshall Islands (Nitta and Yanai (1969), and winds with a jet-like structure such as the easterlies over North Africa (Burpee 1972) and the easterlies over the Line Islands (Nitta and Yanai 1969). Winds that resemble a shear layer are shown to be stabilized by divergence, except when the mean flow is marginally unstable, in which case divergence is weakly destabilizing; (see Fig. 1). Jets are shown to be stabilized by divergence

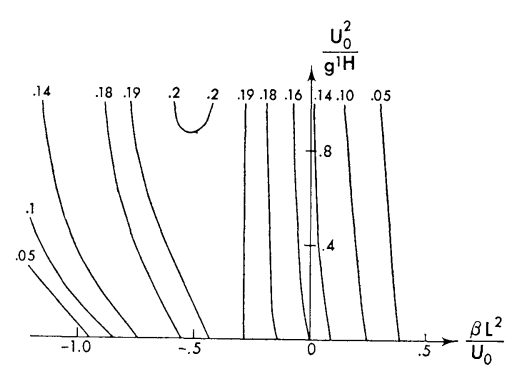

Fig. 2 Contours of constant growth-rate (in units $U_{o} / L$ ) as a function of the $\beta$ effect and divergence for wavelength $2 \pi L$, for the equatorial jet $U= \pm U_{o}$ $\operatorname{sech}^{2} y / L$. This is essentially the most unstable wavelength. (Positive and negative values of $\beta L^{2} / U_{o}$ correspond to an eastward and westward jet respectively. The results shown here are for the sinuous mode of oscillation, the more unstable of the two possible unstable modes). 
if they are westerly (in which case the $\beta$-effect is also stabilizing). Easterly jets, on the other hand, can be destabilized by both divergence and by the $\beta$-effect. The latter results, which are summarized in Fig. 2, may be relevant to the phenomena described by Burpee (1972, 1974).

\section{Stability of Shear Flow}

Consider a current of intensity $U_{o}$ and width $L$ in the lower layer, of depth $H$, of a two-layer system in which the upper layer is infinitely deep and motionless. (This is the simplest model capable of simulating the effect of divergence.) Let reduced gravity $\mathrm{g}^{\prime}$ denote the acceleration associated with the density difference between the two layers of fluid, Lipps (1963) showed that, if the quasigeostrophic approximations are valid, then the necessary condition for instability is

$$
F=\beta-U_{y y}+U / \lambda^{2} \text { changes sign some- }
$$$$
\text { where in the region of interest. }
$$

Here $\lambda \equiv \sqrt{g^{\prime}} \bar{H} / f_{0}$ is the radius of deformation and $f_{o}$ is the Coriolis parameter. The Marshall Island profiles studied by Nitta and Yanai (1969) may be approximated by expressions of the type

$$
U(y)=-U_{0} \tan h(y / L)-U_{1}
$$

where $U_{o}$ and $U_{1}$ are positive constants. This corresponds to a shear flow imbedded in an easterly drift which has a speed $U_{1}$. For such profiles, $F$ (as defined in 2) satisfies the inequalities

$$
\begin{gathered}
\beta-\frac{4 U_{0}}{3 \sqrt{3} L^{2}}\left(1+-\frac{L^{2}}{\partial \lambda^{2}}\right)^{3 / 2}-\frac{U_{1}}{\lambda^{2}} \leq F \leq \beta \\
+\frac{4 U_{0}}{3 \sqrt{2} L^{2}}\left(1+\frac{L^{2}}{\partial \lambda^{2}}\right)^{3 / 2}-\frac{U_{1}}{\lambda^{2}}
\end{gathered}
$$

It follows that for non-divergent $(\lambda \gg L)$ motion the necessary condition for instability is $U_{o} / L^{2}>$ $(3 \sqrt{ } 3 / 4) \beta$. (The magnitude of the $\operatorname{drift} U_{1}$ is of no consequence. The $\beta$-effect plays a stabilizing role here.) Consider next the situation when the width of the shear zone $L$ is comparable to the radius of deformation $\lambda$ so that divergence is important. (Typically $L \sim 300 \mathrm{~km}$, and $\lambda$ could be as small as $600 \mathrm{~km}$.) It follows from (4) that divergence causes the necessary condition for instability to become less stringent. Divergence also causes the drift $U_{1}$, to affect the stability. If the drift is easterly, it diminishes the importance of the $\beta$-effect, and thus destabilizes the flow. (If the drift is westerly, $U_{1}<0$, then it augments the $\beta$-effect and is stabilizing.) The shear flow (characterized by $U_{o} / L$ ) is always destabilized by divergence, even if the sign of $U_{o}$ should change.

These inferences concerning the destabilizing role of divergence are only valid close to the curve of neutral stability (where the growth-rates of unstable waves are nearly zero). To determine how divergence affects the stability of the profile (3) when growth-rates are not infinitesimal, a stability analysis is necessary. An ageostrophic but linearized two-level model, described by Philander (1976) has been used to calculate growth-rates for the profile (3) with $U_{1}=0$ and with $y=0$ corresponding to the equator. The results are shown in Fig. 1. (Note that a change in the sign of $U_{o}$ leaves these results unaltered.) In the absence of divergence $\left(g^{\prime} H=\infty\right)$ the $\beta$ effect is seen to be stabilizing: growth-rates decrease as $\beta$ increases along the abscissa. In the absence of a $\beta$-effect $\left(L \ll \sqrt{ } \bar{U}_{0}^{-} / \bar{\beta}\right)$ divergence is stabilizing: growth-rates decrease as the importance of divergence increases along the ordinate. However, divergence can be destabilizing once the $\beta$-effect is important $\left(\beta L^{2} / U_{o}>\cdot 4\right)$ This is consistent with the earlier remarks that divergence relaxes the necessary condition for instability. We also learn from Fig. 1 that the $\beta$-effect can be destabilizing when divergence is sufficiently important.

Nitta and Yanai (1969) studied the stability of the surface winds over the Marshall Islands in June 1958 and found the most unstable waves to have a wavelength of $2000 \mathrm{~km}$ and a period of 5 days. These calculations have been repeated with the aforementioned ageostrophic divergent model but the results were found to be practically unchanged. Two factors contribute to this: (1) The appropriate value for the parameter $\beta L^{2} / U_{o}$ is sufficiently small $(<\cdot 4)$ for divergence to be stabilizing if we consider the shear flow in isolation $\left(U_{1}=0\right)$ (2) this stabilization is cancelled by the destabilizing effect of the easterly drift $U_{1}$ in which the shear flow is imbedded. If the value of $\beta L^{2} / U_{o}$ had been larger, then the net effect of divergence would have been to destabilize the flow.

\section{Stability of Jets}

The results shown in Fig. 1 are representative of antisymmetric profiles $(U(y)=-U(-y))$. The stability properties of the Bickley jet $U=\operatorname{sech}^{2}$ $y / L$, which can be considered as representative of symmetric profiles $(U(y)=U(-y))$, are shown in Fig. 2. Positive and negative values of the parameter $\beta L^{2} / U_{o}$ correspond to an eastward and 
westward jet respectively. Unlike Fig. 1, Fig. 2 is not symmetric about the $\beta=0$ axis; the $\beta$-effect influences easterly and westerly currents differently: it stabilizes westerly currents but can destabilize easterly currents provided $U_{o}>2 \beta L^{2}$. (If the flow is divergent, the $\beta$-effect is destabilizing for even smaller values of $U_{o}$.) From Fig. 2 we also learn that whereas divergence stabilizes westerly jets, it can destabilize easterly jets. Hence an easterly jet is much more unstable than a comparable westerly jet.

Burpee $(1974,1972)$ has described waves with a period of 3-5 days that are related to the midtropospheric easterly jet to the south of the Sahara desert. The stability properties of this jet can be expected to be similar to the results of Fig. 2 rather than Fig. 1. (The results of Fig. 2 are for a jet centered on the equator. Displacement of the jet from the equator increases the $e$-folding time somewhat; see Philander 1976). A stability analysis of a profile $U=U_{o} \operatorname{sech}^{2} y / L$, where the values of $U_{o}(\sim 15 \mathrm{~m} / \mathrm{sec})$ and $L(\sim 700 \mathrm{~km})$ are appropriate for the mean zonal flow described by Burpee (1972), gives results in good agreement with the observations: unstable waves with a period of about 4 days and a wave-length of approximately $4000 \mathrm{~km}$. (For these values of $U_{o}$ and $L_{1}, \beta L^{2} / U_{o}=-.67$.) The values for the period and most unstable wavelength are rela- tively insensitive to the value of the effective Richardson number $g^{\prime} H / U_{o}{ }^{2}$. The $e$-folding time, however, is sensitive to the value of this parameter especially if the value of $\beta L^{2} / U_{o}$ is as low as .67. To assign a value to $g^{\prime} H$ is obviously a difficult matter. We therefore merely point out that the $e$-folding time varies from 4 days (when the flow is non-divergent) to 2.8 days when $g^{\prime} H / U_{o}^{2}=1$. A study of the energetics of a divergent unstable jet (see Philander 1976) shows that both the horizontal and vertical Reynolds' stresses transfer kinetic energy from the mean flow to the eddies. Conversion of potential energy is unimportant.

\section{References}

Burpee, R. W. (1972): The origin and structure of easterly waves in the lower troposphere of North Africa. J. Atmos. Sci., 29, 77-90.

(1974): Characteristics of North African easterly waves during the summers of 1968 and 1969. J. Atmos. Sci., 31, 1556-1570.

Lipps, F. B. (1963): Stability of jets in a divergent barotropic fluid. J. Atmos. Sci., 20, 120-129.

Nitta, T., and Yanai, M. (1969): A note on the barotropic instability of the tropical easterly current. J. Met. Soc. Japan, 47, 127-130.

Philander, S. G. H. (1976): Instabilities of zonal equatorial currents. Journal of Geophysical Research, 81, 3725-3735.

\title{
熱帯偏東風の安定性についてのノート
}

\author{
S. G. H. Philander \\ Geophysical Fluid Dynamics Programme \\ プリンストン大学, プリンストン，アメリカ合衆国
}

